\title{
Design of Synthetic Quasi-TEM Transmission Line for CMOS Compact Integrated Circuit
}

\author{
Meng-Ju Chiang, Student Member, IEEE, Hsien-Shun Wu, Member, IEEE, and \\ Ching-Kuang C. Tzuang, Fellow, IEEE
}

\begin{abstract}
This paper presents the design guidelines of the synthetic quasi-TEM transmission line (TL) based on standard 0.18- $\mu \mathrm{m}$ one-poly six-metal complementary metal-oxide-semiconductor (CMOS) technology. The synthetic quasi-TEM TL, also called the complementary-conducting-strip transmission line (CCS TL), is composed of five structural parameters to synthesize its guiding characteristics. Twenty-four designs of CCS TL are reported, with the following unique attributes. First, a characteristic impedance range of 8.62-104.0 $\Omega$ is yielded. Second, the maximum value of the slow-wave factor is 4.79 , representing an increase of $139.5 \%$ over the theoretical limit of the quasi-TEM TL. Third, the ratio of the area of the CCS TL to its corresponding quality factor ( $Q$ factor) can help to estimate the cost of the loss for the circuit miniaturizations. Additionally, the important CMOS manufacturing of metal density is for the first time involved in the reported TL designs. By following the proposed design methodologies, a practical design example of a $K a$-band CMOS rat-race hybrid is reported and experimentally examined in detail to reveal the feasibility of the proposed design guidelines to synthesize the CMOS CCS TL. The chip size without contact pads is $420.0 \mu \mathrm{m}$ $\times 540.0 \mu \mathrm{m}$. The measured loss and isolation of the hybrid at 36.3 $\mathrm{GHz}$ are 3.84 and $58.0 \mathrm{~dB}$, respectively.
\end{abstract}

Index Terms-Complementary metal-oxide-semiconductor (CMOS), hybrid, quasi-TEM, thin-film microstrip (TFMS), transmission line (TL).

\section{INTRODUCTION}

C OMPLIMENTARY metal-oxide-semiconductor(CMOS) technology promises a higher level of integration and lower cost than the III-V compounds, enabling the production of multifunction RF transceivers and RF system-on-chip (SOC) designs [1]-[5]. These multifunction circuits on silicon generally adopted lumped or lumped- distributed equivalence, which is faced with not only operation at ever-increasing frequencies, but also saving power consumption and chip area when the processes are scaled down. On the other hand, the transmission line (TL) frameworks, such as microstrip (MS) and coplanar waveguide (CPW), have gained popularity in CMOS RF circuit designs [6]-[12]. Moreover, such TLs can be made to establish a 3-D monolithic microwave integrated circuit (MMIC) in order to save chip area [13]-[19]. The concept of the synthetic quasi-TEM TL was recently reported and successfully employed to miniaturize RF integrated circuits [20]-[23]. Such

Manuscript received May 15, 2007. This work was supported by the National Science Council of Taiwan, R.O.C., under Grant NSC 96-2752-E-002009-PWE and Grant NSC 95-2221-E-002-084-MY2.

The authors are with Graduate Institute of Communication Engineering, National Taiwan University, Taipei 106, Taiwan, R.O.C. (e-mail: cktzuang@cc.ee. ntu.edu.tw).

Digital Object Identifier 10.1109/TMTT.2007.910089 successes are mainly due to an efficient meandering of the TL to achieve the highest degree of integration.

Extensive studies indicate that the synthetic quasi-TEM TL, also called the complementary-conducting-strip transmission line (CCS TL), has better guiding properties than those of the conventional MS when the signal trace is meandered in a 2-D plane [21]. Following the same concept of miniaturization, this study focuses on the design of the CCS TL using standard $0.18-\mu$ m one-poly six-metal (1P6M) CMOS technology, which is available from most standard silicon foundry services in the world. Fig. 1 illustrates two approaches to designing a CMOS CCS TL. As shown in Fig. 1, the signal traces of the CCS TL are meandered by obeying two basic rules. First, the length of the signal trace is fixed. The length is defined as $270.0 \mu \mathrm{m}$ in this study. Second, the CCS TL is meandered by at least four bends in the square area. By following the winding course defined above, the CCS TLs are designed for the characteristics impedance $\left(Z_{c}\right)$ of $88.1,50.7$, and $22.7 \Omega$. The CCS TLs in the bottom row of Fig. 1 are designed by referring to the concept of thin-film microstrip (TFMS), which is regarded as a special limiting case. Although all the limiting cases are meandered with a line space of $2.0 \mu \mathrm{m}$, which is the minimum value defined in this study, the corresponding area increases when $Z_{c}$ decreases. For example, the area of 22.7- $\Omega$ CCS TL in Fig. 1(f) is $10152.0 \mu \mathrm{m}^{2}$, representing 10.25 occurrences of $88.1-\Omega$ CCS TL in Fig. 1(d). However, the CCS TL is designed with stacked metal by manipulating the advantage of multilayer CMOS technology. Fig. 1(a) and (c) shows the 22.7- and 88.1- $\Omega$ CCS TLs, which can be designed with areas of 6750.0 and $1019.7 \mu \mathrm{m}^{2}$, respectively. Conversely, the quality factor ( $Q$ factor) of the 88.1- $\Omega$ CCS TL in Fig. 1(a) at $10.0 \mathrm{GHz}$ is 1.97, which is $6.8 \%$ lower than that in Fig. 1(d). The slow-wave factor (SWF) of the 22.7- $\Omega$ CCS TL in Fig. 1(b) at $10.0 \mathrm{GHz}$ is 2.67 , which is $33.5 \%$ higher than the theoretical limit of the quasi-TEM TL. Furthermore, by applying the stacked metal to the designs of 22.7- and 50.7- $\Omega$ CCS TLs in Fig. 1(a) and (b), two CCS TLs automatically meet the metal density requirement without inserting additional dummy metals. The metal density, which denotes the ratio of the total metal layout area to the $\mathrm{TL}$ area, is strongly required by the foundry to manage the variation of chemical-mechanical polishing (CMP) in the wafer manufacture, maintaining the wafer yield and design reliability [24]. The design approaches, which lead to different guiding characteristics of the CCS TL, are extensively investigated in Section III after the validity check of the full-wave EM simulation, which is presented in Section II for extracting the guiding characteristics of the CMOS CCS TL. Section IV reports a 


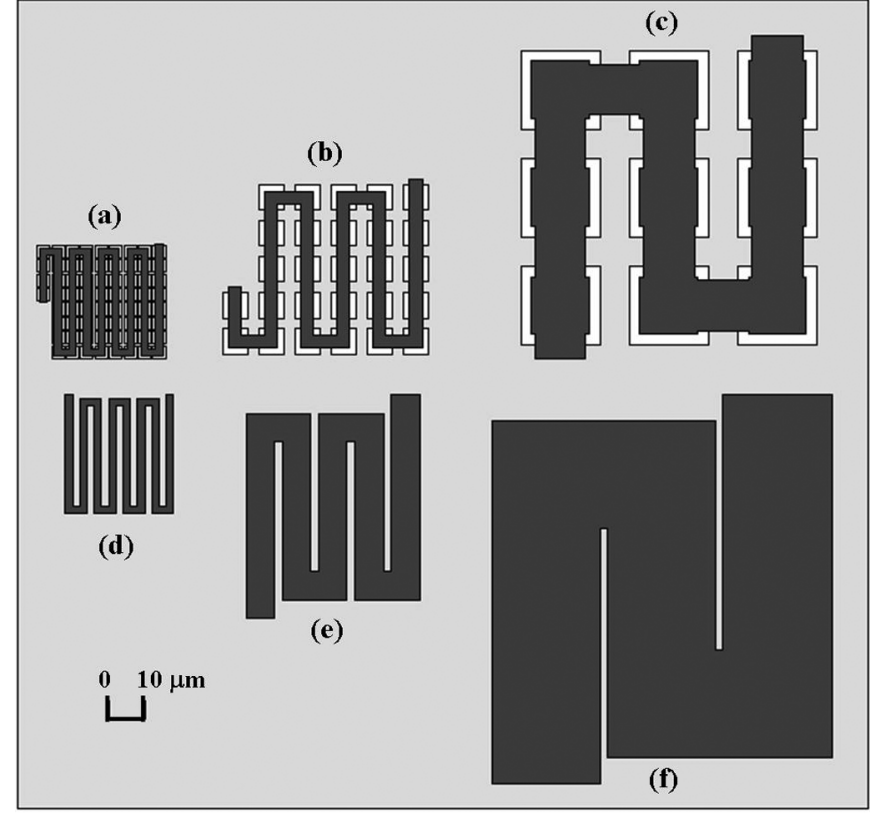

Fig. 1. CMOS synthetic quasi-TEM TL. The length of all TLs is $270.0 \mu \mathrm{m}$. (a) $Z_{c}=88.1 \Omega$. (b) $Z_{c}=50.7 \Omega$. (c) $Z_{c}=22.7 \Omega$. (d) $Z_{c}=88.1 \Omega$. (e) $Z_{c}=50.7 \Omega$.(f) $Z_{c}=22.7 \Omega$

practical example of a $K a$-band rat-race hybrid realized by the CCS TL, based on the design guidelines summarized at the end of Section III to reveal the superior performance in terms of low loss and compact area of the CMOS integration. Finally, Section V presents a brief summary.

\section{CMOS MulTILAYER SYNTHETIC QUASI-TEM TL: CCS TL}

The proposed CCS TL is constructed by the unit cell on the silicon substrate. As shown in Fig. 2(a), the unit cell, whose dimensions are much smaller than the guiding wavelength at the operating frequency, is the smallest element in the TL. The signal trace is composed of a central patch and four connecting arms, with the latter used to connect adjacent cells. Fig. 2(a) only displays two arms for simplicity. However, the central patch and mesh ground plane can be constructed by using solid vias to link metals in a multilayer structure. As shown in Fig. 2(b), the thickness of the mesh ground plane is increased by stacking $M_{1}$ to $M_{5}$ in order to decrease the series resistance of the TL, thus enhancing the $Q$ factor of the CCS TL [21]. The unit cell in Fig. 2(a) shows a periodicity of $P$, and alternately combines two types of TLs shown in Fig. 2(b) and (c) to form a quasi-TEM TL. Fig. 2(b) displays a cross-sectional view of the $A-A^{\prime}$ cut, and clearly shows the well-known MS structure, which is locally a capacitive region from the circuit point of view. In contrast, Fig. 2(c) displays an MS with a tuning septa [25], which can be regarded as an elevated CPW [26], and is a high-impedance inductive region alongside the $B-B^{\prime}$ cut. Both microminiaturized guiding frameworks support the quasi-TEM mode, alternating to guide the electromagnetic (EM) energy, thus allowing arbitrary syntheses of the quasi-TEM TL with required characteristic impedance [21]. The central patch with a dimension $W$ and the mesh ground plane of an inner slot with a dimension

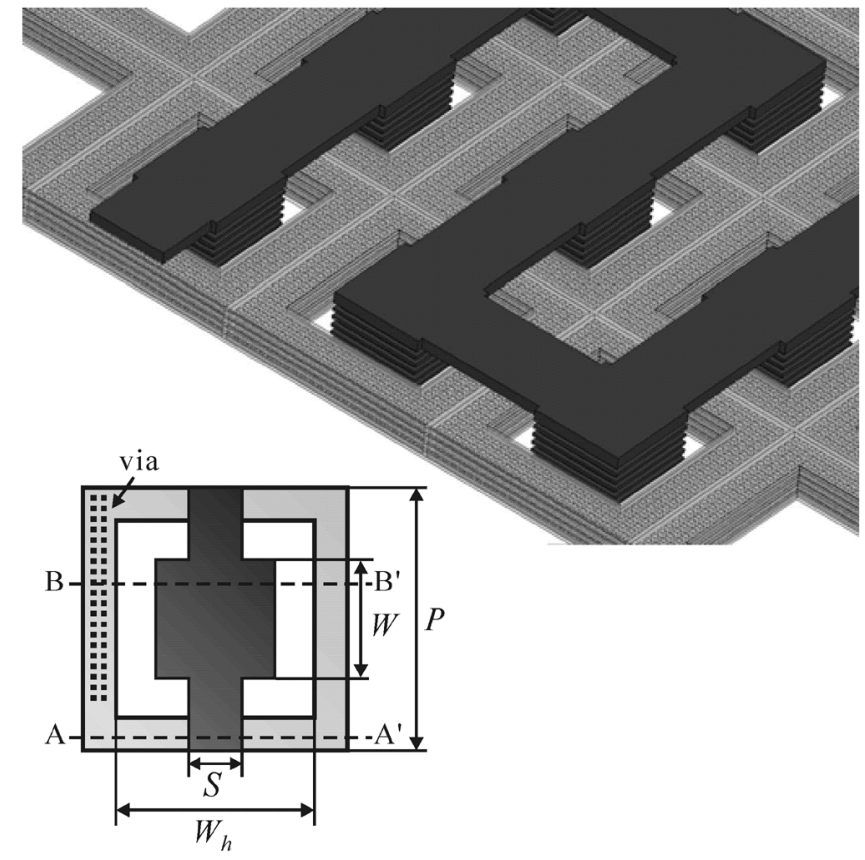

(a)

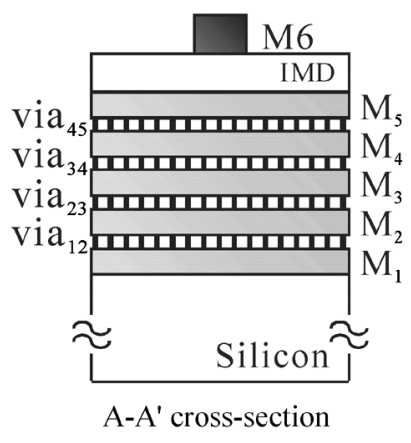

(b)

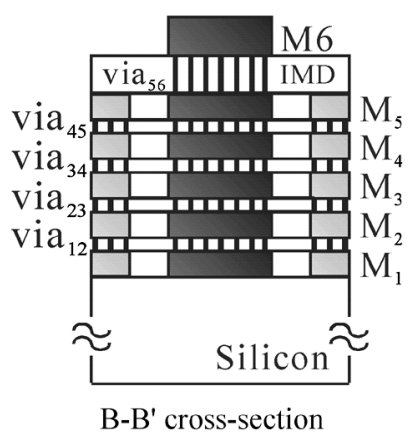

(c)
Fig. 2. CMOS CCS TL. (a) 3-D view. (b) Cross-sectional view of $A-A^{\prime}$ cut in (a). (c) Cross-sectional view of $B-B^{\prime}$ cut in (a).

$W_{h}$ form the complementary conducting surfaces. The term $S$ denotes the width of the connecting arm, thus forming the so-called CCS TL. If $S=W$ and $W_{h}=0$, then the CCS TL is regarded as the conventional TFMS, forming a special limiting case in Fig. 2. Furthermore, the values of the structural parameters, namely, $P, W_{h}, S, W$, and the number of metal layer are restricted by the capability of the CMOS technology, which defines the minimum and maximum values of line space, linewidth, and number of metal layers. Hence, the proposed CCS TL design is scalable by following the continuing improvement of the semiconductor technology.

Fig. 3(a) shows a chip photograph of a practical design example of the proposed CCS TL in Fig. 2(a). The signal trace is realized by $M_{6}$, and the mesh ground plane is made of metal layers from $M_{1}$ to $M_{5}$. As shown in Fig. 3(a), the prototype is designed with the following structural parameters: $W=5.0 \mu \mathrm{m}, S=4.0 \mu \mathrm{m}, P=30.0 \mu \mathrm{m}$, and $W_{h}=28.0 \mu \mathrm{m}$. The relative dielectric constants of the inter-media-dielectric (IMD) and silicon substrate are 4.0 and 11.9, respectively. The thickness and conductivity of the silicon substrate are $482.6 \mu \mathrm{m}$ 


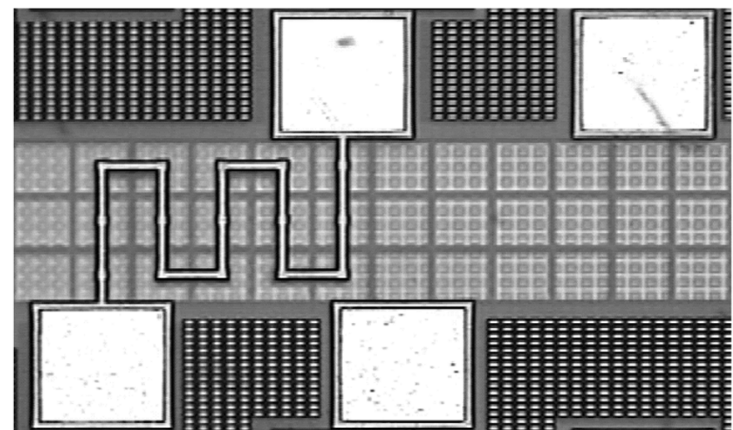

(a)

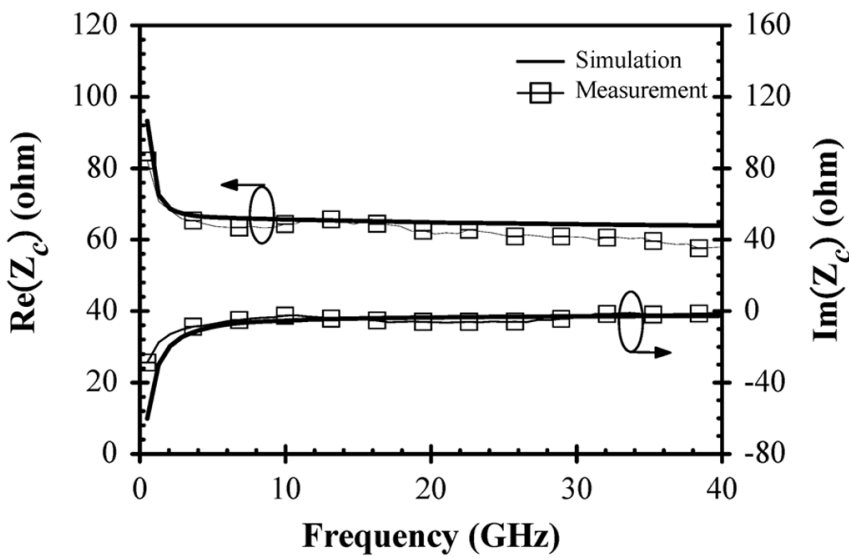

(b)

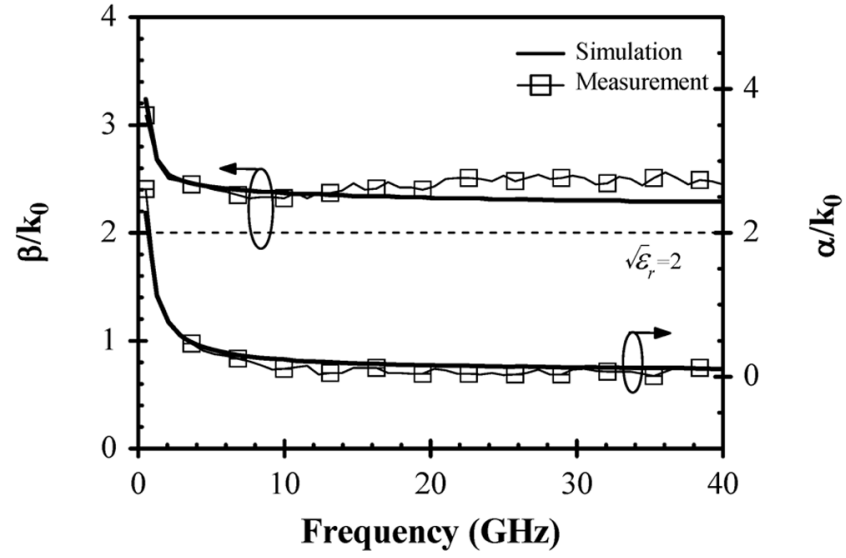

(c)

Fig. 3. Validity checks of the simulated results of CCS TL against measurement. (a) Chip photograph of the prototype fabricated by $0.18-\mu \mathrm{m} 1 \mathrm{P} 6 \mathrm{M} \mathrm{CMOS}$ technology. (b) Complex characteristic impedance of prototype. (c) Complex propagation constant of prototype. Structural and material parameters are $W=$ $5.0 \mu \mathrm{m}, S=4.0 \mu \mathrm{m}, P=30.0 \mu \mathrm{m}$, and $W_{h}=28.0 \mu \mathrm{m}$. The relative dielectric constants of the IMD and silicon substrate are 4.0 and 11.9 , respectively. The thickness and conductivity of the silicon substrate are $482.6 \mu \mathrm{m}$ and 11.0 S/m, respectively. The thickness and resistivity of $M_{6}$ layer are $2.0 \mu \mathrm{m}$ and $37 \mathrm{~m} \Omega / \mathrm{sq}$, respectively. The thickness and resistivity of the layers $M_{1}-M_{5}$ are $0.55 \mu \mathrm{m}$ and $79 \mathrm{~m} \Omega / \mathrm{sq}$, respectively.

and $11.0 \mathrm{~S} / \mathrm{m}$, respectively. The thickness and resistivity of $M_{6}$ layer are $2.0 \mu \mathrm{m}$ and $37 \mathrm{~m} \Omega / \mathrm{sq}$, respectively. The thickness and resistivity of the layers $M_{1}-M_{5}$ are $0.55 \mu \mathrm{m}$ and $79 \mathrm{~m} \Omega / \mathrm{sq}$, respectively. The characteristics of the CCS TL are gained from the on-wafer measurements. The two-port $S$-parameters of the CCS TL are measured after the short-open-load-thru (SOLT) calibration procedures have been carried out to eliminate the parasitics of the signal-ground (SG) pads. After the two-port $S$-parameters are obtained, the complex propagation constant $(\gamma=\alpha+j \beta$, where $\alpha$ is the attenuation constant and $\beta$ is the phase constant) and characteristic impedance $\left(Z_{c}\right)$ are extracted by the well-documented procedures in [21]. Parallel to the on-wafer measurements, the CCS TL shown in Fig. 3(a) is also theoretically examined by Ansoft's commercial software package High Frequency Structure Simulator (HFSS) with the structural and material parameters mentioned above. The simulated results are also compared to those of the extracted results based on the measurements to verify the validity of full-wave EM simulations.

Fig. 3(b) and (c) shows the comparisons. The maximum deviation of $5.7 \%$ in the real part of characteristics impedance $\left(Z_{c}\right)$ is achieved in the range from 5.0 to $30.0 \mathrm{GHz}$. Two imaginary parts of $Z_{c}$ are nearly identical. Furthermore, the measured normalized phase constants, denoted by $\beta / k_{0}$, indicate a maximum difference of $8.0 \%$, as opposed to the HFSS simulations, and two normalized attenuation constants are nearly identical. The normalized phase constant is 2.37 at $10.0 \mathrm{GHz}$, which is higher than the theoretical limit $\sqrt{\varepsilon_{r}}$ of the quasi-TEM TL. The value $\varepsilon_{r}$ is the relative dielectric constant of the IMD. Two sets of curves in Fig. 3(b) and (c) shows excellent agreement in the range from 5.0 to $30.0 \mathrm{GHz}$, confirming the validity of the on-chip CCS TL characteristics using full-wave EM simulations. Section III presents the analysis of the CMOS CCS TL shown in Fig. 2(a) with various structural parameters via Ansoft's commercial software HFSS. The design guidelines for CCS TL to synthesize the specific guiding properties are also summarized based on the extensive EM simulations.

\section{DESIGN OF MEANDERED CMOS CCS TLS}

\section{A. Simulation Setups for Theoretical CCS TL Designs}

The design of the CCS TL by using standard $0.18-\mu \mathrm{m} 1 \mathrm{P} 6 \mathrm{M}$ CMOS technology is focused upon here. The material parameters, including the substrate thicknesses and relative dielectric constant, for the HFSS simulations, are set up by following the definitions reported in Section II. Furthermore, $M_{6}$ of all TLs in this study are designed with the maximum and minimum linewidths of 30.0 and $2.0 \mu \mathrm{m}$, respectively. The minimum line space of $M_{6}$ is $2.0 \mu \mathrm{m}$. Both the minimum linewidth and line space for layers $M_{1}-M_{5}$ are $0.5 \mu \mathrm{m}$. It is to be noted that the design rules for all these metal layers mentioned above conform to the standard foundry rules defined by most manufacturers. Conversely, before performing the HFSS simulations, the CCS TL is meandered by following two basic rules reported in Section I. The physical length of the TL is $270.0 \mu \mathrm{m}$, and the TL is meandered by at least four bends in each square area. The guiding properties of the CMOS CCS TL at $10.0 \mathrm{GHz}$, namely, characteristic impedance $\left(Z_{c}\right), \mathrm{SWF}$, and $Q$ factor are extracted by the same procedures reported in Section II. The SWF is defined as the normalized phase constant $\left(\beta / k_{0}\right)$ of the CCS TL, and the $Q$ factor is the ratio of the phase constant to twice of the attenuation constant. Table I summarizes the extracted results of varying the corresponding structural parameters $P, W_{h}, S, W$, and the metal layers for discussions in the following sections. 
TABLE I

REFERENCE DESIGNS OF 10.0-GHz CCS TLs Using STANDARD 0.18- $\mu \mathrm{m}$ 1P6M CMOS TECHNOLOGY

\begin{tabular}{|c|c|c|c|c|c|c|c|c|c|c|c|c|c|c|c|c|c|c|}
\hline \multirow{2}{*}{ Type } & \multirow{2}{*}{$\begin{array}{c}\operatorname{Re}\left(Z_{c}\right) \\
(\Omega) \\
\end{array}$} & \multirow{2}{*}{$\begin{array}{c}P \\
(\mu \mathrm{m})\end{array}$} & \multirow{2}{*}{$\begin{array}{c}W_{h} \\
(\mu \mathrm{m})\end{array}$} & \multirow{2}{*}{$\begin{array}{c}S \\
(\mu \mathrm{m})\end{array}$} & \multirow{2}{*}{$\begin{array}{c}W \\
(\mu \mathrm{m})\end{array}$} & \multicolumn{6}{|c|}{ Signal Trace } & \multicolumn{5}{|c|}{ Mesh Ground } & \multirow{2}{*}{$Q$} & \multirow{2}{*}{ SWF } \\
\hline & & & & & & $\mathrm{M}_{1}$ & $\mathrm{M}_{2}$ & $\mathrm{M}_{3}$ & $\mathrm{M}_{4}$ & $\mathrm{M}_{5}$ & $\mathrm{M}_{6}$ & $\mathrm{M}_{1}$ & $\mathrm{M}_{2}$ & $\mathrm{M}_{3}$ & $\mathrm{M}_{4}$ & $\mathrm{M}_{5}$ & & \\
\hline \multirow{19}{*}{$\begin{array}{l}\text { CCS TL } \\
\left(W_{h} \neq 0\right)\end{array}$} & 104.0 & 30.0 & 29.5 & 2.0 & 2.0 & & & & & & & & & & & & 3.15 & 2.03 \\
\hline & 98.1 & 20.0 & 19.5 & 2.0 & 2.0 & & & & & & & & & & & & 3.11 & 1.82 \\
\hline & 95.6 & 15.0 & 14.5 & 2.0 & 2.0 & & & & & & & & & & & & 3.05 & 1.70 \\
\hline & 92.6 & 10.0 & 9.5 & 2.0 & 2.0 & & & & & & & & & & & & 2.74 & 1.48 \\
\hline & 91.9 & 20.0 & 14.5 & 2.0 & 2.0 & & & & & & & & & & & & 3.50 & 1.82 \\
\hline & 88.1 & 4.0 & 3.5 & 2.0 & 2.0 & & & & & & & & & & & & 1.93 & 0.97 \\
\hline & $71.8^{2}$ & $30.0^{2}$ & $28.0^{2}$ & $4.0^{2}$ & $4.0^{2}$ & & & & & & & & & & & & $4.81^{2}$ & $2.01^{2}$ \\
\hline & 69.2 & 5.0 & 4.0 & 2.0 & 2.5 & & & & & & & & & & & & 2.27 & 1.24 \\
\hline & 65.8 & 30.0 & 28.0 & 4.0 & 4.0 & & & & & & & & & & & & 5.19 & 2.35 \\
\hline & $64.7^{1}$ & $30.0^{1}$ & $28.0^{1}$ & $4.0^{1}$ & $5.0^{1}$ & & & & & & & & & & & & $5.04^{1}$ & $2.37^{1}$ \\
\hline & 50.7 & 10.0 & 7.0 & 3.5 & 3.5 & & & & & & & & & & & & 3.17 & 1.97 \\
\hline & 36.0 & 15.0 & 10.0 & 6.0 & 9.0 & & & & & & & & & & & & 3.62 & 2.15 \\
\hline & 34.1 & 15.0 & 10.5 & 6.0 & 6.0 & & & & & & & & & & & & 3.38 & 2.37 \\
\hline & 35.1 & 30.0 & 20.0 & 10.0 & 10.0 & & & & & & & & & & & & 4.97 & 2.51 \\
\hline & 34.3 & 30.0 & 25.0 & 10.0 & 12.0 & & & & & & & & & & & & 4.77 & 2.64 \\
\hline & 30.7 & 30.0 & 26.0 & 10.0 & 14.0 & & & & & & & & & & & & 4.63 & 2.76 \\
\hline & 22.7 & 30.0 & 22.0 & 14.0 & 16.0 & & & & & & & & & & & & 4.03 & 2.67 \\
\hline & 12.4 & 10.0 & 5.0 & 4.0 & 4.0 & & & & & & & & & & & & 2.01 & 4.79 \\
\hline & 8.62 & 30.0 & 17.0 & 14.0 & 16.0 & & & & & & & & & & & & 1.97 & 4.18 \\
\hline \multirow{5}{*}{$\begin{array}{c}\text { CCS TL } \\
\left(W_{h}=0, S=W\right) \\
\text { or } \\
\text { TFMS }\end{array}$} & 88.1 & & 0 & 2.0 & 2.0 & & & & & & & & & & & & 2.07 & 0.96 \\
\hline & 69.2 & & 0 & 4.0 & 4.0 & & & & & & & & & & & & 2.93 & 1.09 \\
\hline & 50.7 & & 0 & 8.0 & 8.0 & & & & & & & & & & & & 3.58 & 1.21 \\
\hline & 35.8 & & 0 & 16.0 & 16.0 & & & & & & & & & & & & 4.21 & 1.49 \\
\hline & 22.7 & & 0 & 30.0 & 30.0 & & & & & & & & & & & & 4.42 & 1.62 \\
\hline
\end{tabular}

design parameters and extracted results in Fig. 3.

${ }^{2}$ design parameters applied in Fig. 7(a).

The metal layer, which is applied to the CCS TL design, is highlighted in Table I, which also shows the corresponding metal density, defined as the ratio of the total metal layout area to the TL area. The metal layers with the metal density below and above $30.0 \%$ are shown in gray and black, respectively. This work on CMOS TL design is the first to take the process issue of the metal density into consideration. Such a process issue, which is specifically defined by the manufacturer, dominated the yield of the CMOS circuit.

\section{B. Special Limiting Designs of CCS TL}

The CCS TL is identical to the conventional TFMS when $W_{h}=0$ and $S=W$. Conversely, as indicated in [27, Fig. 5], the $Q$ factor of the TFMS significantly decreases if the effective thickness between the signal trace and ground plane decreases. Hence, the CCS TL in this category applied $M_{1}$ to the ground plane and $M_{6}$ to the signal trace to achieve low loss. However, the drawback of the low-loss design is that the metal densities of the rest of the metal layers, from $M_{2}$ to $M_{5}$, are zero. Additional chip area is stipulated to accommodate the dummy metal inserts. Additionally, due to the limiting designs of the CCS TL reported in Table I, the line space of $M_{6}$ is set to $2.0 \mu \mathrm{m}$ to minimize the area of the layout. Based on the closed-form expressions of the limiting designs of the CCS TL, $Z_{c}$ and complex propagation constant $\gamma(\gamma=\alpha+j \beta)$ are determined by the linewidth once the material parameters and the thickness between the signal trace and ground plane are fixed [28]. Therefore, as indicated in Table I, characteristic impedance $\left(Z_{c}\right)$ increased from 22.7 to $88.1 \Omega$ when the linewidth of $M_{6}$ decreased from 30.0 to $2.0 \mu \mathrm{m}$. It is to be noted that 30.0 and $2.0 \mu \mathrm{m}$ are the maximum and minimum linewidths defined in Section III-A, which limit the $Z_{c}$ syntheses of the CCS TL. Moreover, at $10.0 \mathrm{GHz}$, the $Q$ factor of the $88.1-\Omega$ CCS TL is 2.07 , which is over $100 \%$ lower than that of the $22.7-\Omega$ CCS TL. The SWF of five speciallimiting designs is below 2 , which is the theoretical limit of the quasi-TEM TL on the substrate with a relative dielectric constant of 4.

\section{CCS TL With $W_{h} \neq 0$}

The CCS TLs with $W_{h} \neq 0$ in Table I show the following design characteristics. The characteristic impedance $\left(Z_{c}\right)$ can be elevated above $88.1 \Omega$ simply by decreasing the ratio of $P$ to $W_{h}$. As shown in Fig. 2(c), $P$ and $W_{h}$ determine the effective area of the high-impedance region in CCS TL. Therefore, $Z_{c}$ can be raised by adjusting $P$ and $W_{h}$ without varying $S$ and $W$, which determine the effective linewidth of the CCS TL. Conversely, to synthesize $Z_{c}$ below $22.7 \Omega$, the metal layer of central patch is vertically extended from $M_{6}$ to $M_{1}$. Such an extension enlarges the overlapping area between the signal trace and ground plane, resulting in an increase of capacitance 


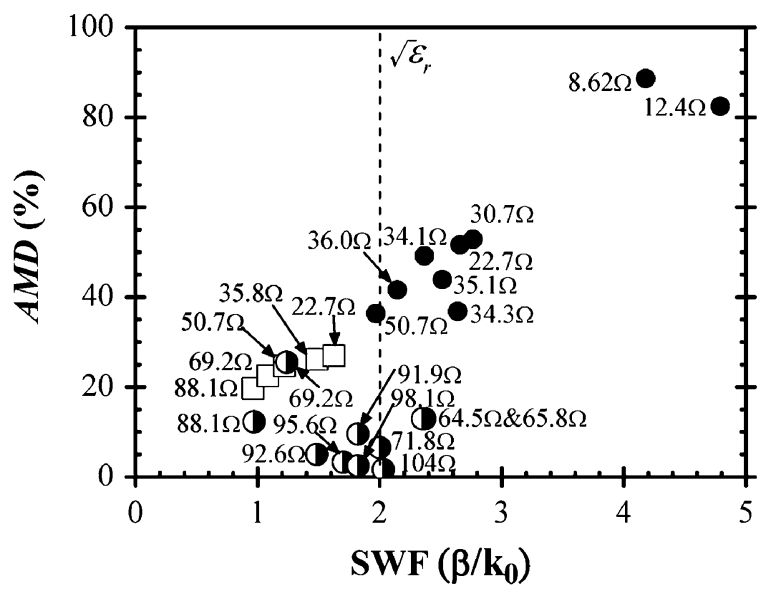

Fig. 4. AMD against the SWF for CCS TLs at $10.0 \mathrm{GHz}$.

per unit length of the CCS TL. By applying the design guidelines to $Z_{c}$ syntheses, the value of $Z_{c}$ ranges from 8.62 up to $104.0 \Omega$, showing a $Z_{c}$ ratio of $12.06(104.0 \Omega / 8.62 \Omega)$. This ratio is significantly wider than that of the TFMS designs reported in Section III-B.

Moreover, the SWF of the CCS TL can be raised by the following two design guidelines. The first guideline is to reduce the ratio of $P$ to $W_{h}$. This approach is applied to designing the CCS TL with $Z_{c}$ from 88.1 to $104.0 \Omega$. As indicated in Table I, the SWF increased from 0.97 to 2.03 when $P / W_{h}$ is reduced from 1.14 to 1.02 . The second approach is to adopt stacked metal to realize the central patch or mesh ground of the CCS TL. In Table I, all the designs of the CCS TL with $Z_{c}$ below $50.0 \Omega$, designed by following the second approach mentioned above, have SWF values exceeding the theoretical limit of the quasi-TEM TL. Meanwhile, these CCS TLs meet the $30.0 \%$ metal density requirement for all metal layers and need no additional chip area for filling dummy metal, attaining true miniaturization. To the best of the authors' knowledge, the proposed design is the first to comply with CMOS metal density rules for designing the CMOS TL. To discuss these two design approaches in details, Fig. 4 plots the SWF at $10.0 \mathrm{GHz}$ against average metal density for all the CCS TL designs in Table I. The average metal density (AMD), as follows in (1), indicates the average value of the metal densities for all six metal layers:

$$
\mathrm{AMD}=\frac{\sum_{i=1}^{6}\left(\frac{\text { Total metal area at } M_{i} \text { layer }}{\text { Area of transmission line }}\right) \times 100 \%}{6} .
$$

As shown in Fig. 4, the CCS TLs, which are marked by symbols in hollow squares $(\square)$, represent the special limiting designs presented in Section III-B. Additionally, the filled circles (-) indicate that the metal densities of all six metal layers in the CCS TL design are higher than or equal to $30.0 \%$. The half-filled circles (O) mean at least one metal layer with metal density less than $30.0 \%$. The quantity adjacent to the symbols represents the $Z_{c}$ values of the CCS TL design listed in Table I.

As shown in Fig. 4, the CCS TLs, which are designed by decreasing the ratio of $P / W_{h}$, show that the SWF increases from 0.97 to 2.03 , while the AMD falls from $12.2 \%$ to $1.7 \%$. The designs based on the first approach do not easily meet the required $30.0 \%$ metal density. Conversely, in the designs following the

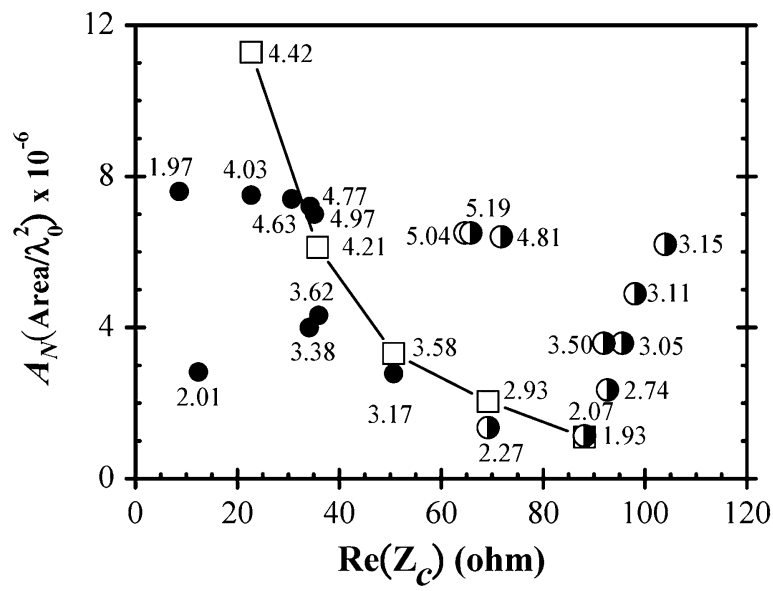

Fig. 5. Normalized area $\left(A_{N}\right)$ against real part of the characteristic impedance $\left(Z_{c}\right)$ for $270.0-\mu \mathrm{m}$-long CCS TLs at $10.0 \mathrm{GHz}$. The symbols in Fig. 5 are identical to those shown in Fig. 4.

second approach, the corresponding SWF increases from 1.97 to 4.79, and meanwhile the AMD also increases from $36.3 \%$ to $82.5 \%$. This trend indicates that the second approach can realize a CCS TL with high SWF, and that the CCS TL design can easily meet the metal density requirement, enabling successful circuit miniaturization. The design approaches for the CCS TL, which can synthesize a TL with various structure parameters, reveal the fundamental modifications to the design of the CMOS TL. Furthermore, a glance back at Fig. 1 reveals that the CCS TLs can be realized in different areas for the same $Z_{c}$, thus attaining different $Q$ factors. Hence, Section III-D is devoted to the discussion of the CCS TL designs with different area.

\section{Area-Influence Loss (AL) of CCS TL}

Fig. 5 plots the normalized area $\left(A_{N}\right)$ versus the characteristic impedance $\left(Z_{c}\right)$ for the CCS TL designs listed in Table I. The term $A_{N}$, defined by (2) as follows, represents the ratio of the total occupying area of the meandered CCS TL with a fixed length of $270.0 \mu \mathrm{m}$ to the square of guided wavelength in free space at $10.0 \mathrm{GHz}$ :

$$
A_{N}=\frac{\text { Area }}{\left(\lambda_{0}\right)^{2}}=\frac{A \cdot\left(f_{0}\right)^{2}}{c^{2}}
$$

where $c$ denotes the velocity of light in free space and $f_{0}$ is the operating frequency. As shown in Fig. 5, the quantity adjacent to the symbols is the $Q$ factor of the CCS TL listed in Table I. The symbols in Fig. 5 are identical to those shown in Fig. 4. As reported in Section III-B, the designs for the special limiting case of the CCS TL, which are meandered by following two basic rules defined in Section I, set the minimum line space at $2.0 \mu \mathrm{m}$ to achieve the smallest compact layout area. As reported in Section III-B, these special limiting cases of the CCS TL are designed according to the closed form in [28]. Therefore, as shown in Fig. 5, the limiting designs of the CCS TL denoted by the symbols in hollow squares $(\square)$, which can form a virtually continuing curve, show the increase of $A_{N}$ inversely proportional to the increase of $Z_{c}$. Such limiting designs are well controlled by only linewidth $(S)$, and can be scaled down with the continuing improvement of semiconductor technology. 
Conversely, the design approaches presented in Section III-C, which can synthesize a wider range of $Z_{c}$ than that of the design approaches in Section III-B and provide multiple designs for one specific $Z_{c}$, lead to different $A_{N}$ distributions. As shown in Fig. 5, to synthesize $Z_{c}$ between 88.1-104.0 $\Omega$, the CCS TL requires a higher $A_{N}$ value than the one predicted by the limiting case designs. This trend shows a reduction of the ratio of $P / W_{h}$ in the CCS TL. Additionally, Fig. 5 shows two group designs with different $A_{N}$ for realizing a 35- $\Omega$ CCS TL. The first design is the CCS TL with $P=30.0 \mu \mathrm{m}$, which achieve the $A_{N}$ approaching that of the limiting case. The second design has $P=15.0 \mu \mathrm{m}$, and results in $A_{N}$ below the predicted value of the limiting case. The $Q$ factor of the CCS TL with $P=30.0 \mu \mathrm{m}$ is approximately 4.87 , which is $39.14 \%$ higher than that of the CCS TL with $P=15.0 \mu \mathrm{m}$. The $Q$ factor of the CCS TL is relatively proportional to the period of the unit cell. This observation reflects the fundamental physical phenomenon of the CCS TL design, which is studied in Fig. 6.

The derivations in $[29, \mathrm{Ch} .7 .4]$ for a rectangular cavity in dominate-mode operation indicate that the conductor loss of the cavity is inversely proportional to its volume [29, Ch. 7.4, pp. 503, eq. (7.48)]. If the width, length, and height of the rectangular waveguide cavity are all identical, then the cavity is regarded as a cubic resonator, and the conductor loss in the resonator is related only to the quantity of the length since all the CCS TLs presented in Table I are designed on the silicon substrate with a fixed thickness, and meandered in a nearly square area, as shown in Fig. 1. Following the concept of the conductor loss in the cubic resonator, this study indicates that the loss of the meandered CCS TL with fixed length is exactly the same as the $\mathrm{AL}$ with the ratio of the square root of the normalized area $\left(A_{N}\right)$ to the $Q$ factor. Additionally, shown in (3) as follows, the AL also can be represented by a function of $f_{0}, A, c$ and the $Q$ factor after some algebraic manipulation:

$$
\mathrm{AL}=\frac{\sqrt{A_{N}}}{Q}=\frac{\sqrt{A / \lambda_{0}^{2}}}{Q}=\frac{\sqrt{A}}{Q \lambda_{0}}=\frac{f_{0} \sqrt{A}}{c Q}
$$

where $A$, which denotes the total occupying area of the meandered CCS TL with a fixed length, is identical to that in (2), $f_{0}$ represents the operating frequency of the TL, and $c$ is the speed of light in free space. As defined in Section I, the length of all the CCS TLs in Table I is set to $270.0 \mu \mathrm{m}$. Therefore, Fig. 6 plots the AL versus characteristic impedance $\left(Z_{c}\right)$ for the CCS TLs in Table I at 5.0, 10.0, 20.0, and $30.0 \mathrm{GHz}$. The values of the parameters at $10.0 \mathrm{GHz}$ are listed in Table I, and the values for 5.0, 20.0 , and $30.0 \mathrm{GHz}$ are obtained by following the same analytical procedures reported in Section II, except for the operating frequency. The definitions of the symbols, which represent various CCS TL designs in Fig. 6, are identical to those in Fig. 4.

Due to the skin effect, the $Q$ factor of the CCS TL is proportional to the square root of the frequency. Thus, the AL of the $50.7-\Omega$ CCS TL at $30.0 \mathrm{GHz}$ is $0.89 \times 10^{-3}$, which is $\sqrt{6}, \sqrt{3}$, and $\sqrt{1.5}$ times those at 5.0,10.0, and $20.0 \mathrm{GHz}$, respectively. Such physical trends also can be observed at different CCS TL designs with characteristic impedance $\left(Z_{c}\right)$ from 22.7 to $88.1 \Omega$.

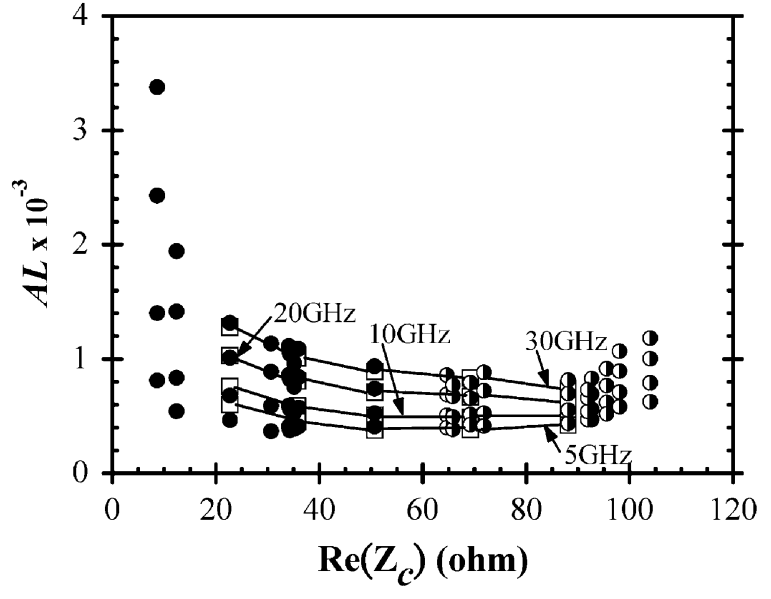

Fig. 6. Characteristic impedance $\left(Z_{c}\right)$ against the AL for CCS TLs with a length of $270.0 \mu \mathrm{m}$. The symbols in Fig. 6 are identical to those shown in Fig. 4.

TABLE II

DESIGN GUIDELINES OF CMOS CCS TL

\begin{tabular}{ccccc}
\hline \hline & $P / W_{h} \uparrow$ & $S \uparrow$ & $W_{h} / W \uparrow$ & \# Metal Layer $\uparrow$ \\
\hline $\mathrm{Z}_{c}$ & $\downarrow$ & $\downarrow$ & $\downarrow$ & $\downarrow$ \\
$Q$ & $\uparrow$ & $\uparrow$ & $\uparrow$ & $\uparrow$ \\
$\mathrm{SWF}$ & $\downarrow$ & $\uparrow$ & $\downarrow$ & $\uparrow$ \\
$A M D$ & $\uparrow$ & $\uparrow$ & $\uparrow$ & $\uparrow$ \\
\hline \hline
\end{tabular}

Furthermore, a close observation of the multiple CCS TL designs with a specific $Z_{c}$ from 22.7 to $88.1 \Omega$ indicates that the corresponding AL value are nearly identical to each other at the same operating frequency, showing a constant ratio between the square root of the normalized area $\left(A_{N}\right)$ to the $Q$ factor in different designs. This result confirms the observation of the two design approaches for the 35- $\Omega$ CCS TL in Fig. 5. The CCS TL can be designed with a relatively high value of $A_{N}$ to raise the corresponding $Q$ factor. Consequently, as shown in Fig. 5, the 22.7- $\Omega$ CCS TL can be designed with $A_{N}=7.5 \times 10^{-6}$ for a $Q$ factor of 4.03, or designed with $A_{N}=11.28 \times 10^{-6}$ for a $Q$ factor of 4.42. Similarly, the 69.2- $\Omega$ CCS TL can be designed with $A_{N}=1.35 \times 10^{-6}$ for a $Q$ factor of 2.27 , or designed with $A_{N}=2.04 \times 10^{-6}$ for a $Q$ factor of 2.93 .

As reported here, the proposed CCS TL provides a high flexibility for synthesizing the desired guiding characteristics. By summarizing the design guidelines reported here, Table II shows the universal trends of synthesizing the desired guiding characteristics by adjusting the structural parameters of the CCS TL. Section IV applies the proposed CCS TL to designing a practical example of the $K a$-band rat-race hybrid by following the design guidelines reported here, demonstrating the capability of the CCS TL.

\section{DESIGN EXAMPLE: $K a$-BAND RAT-RACE HybRID}

Fig. 7(a) shows a 34.3-GHz CMOS rat-race hybrid design incorporating the proposed CCS TLs designed according to the guidelines reported in Section III. The operations of the rat-race 


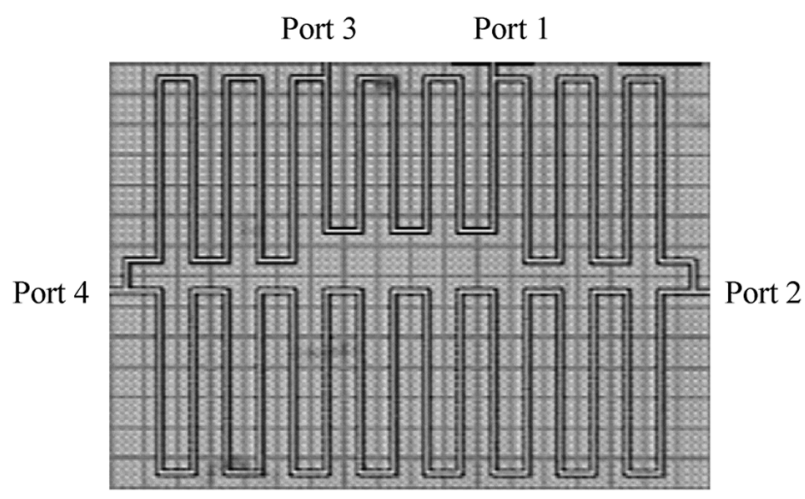

(a)

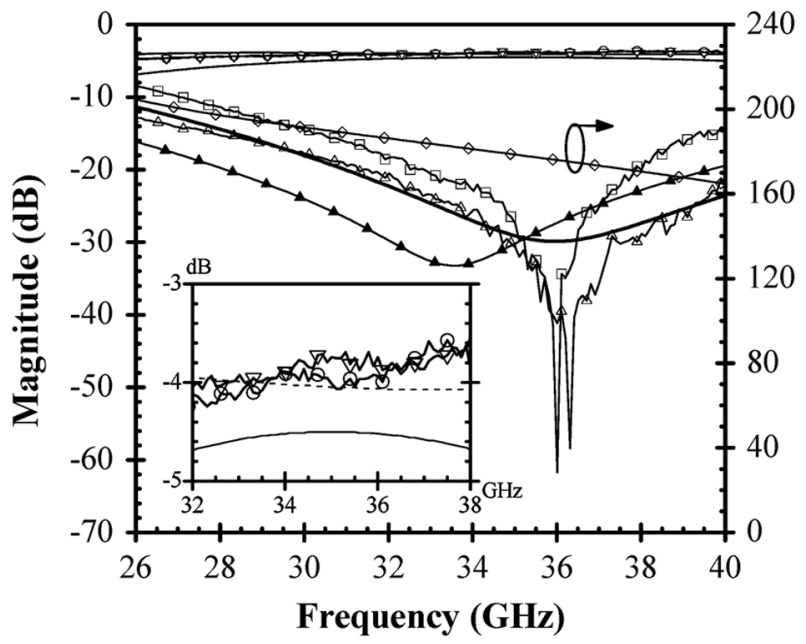

\begin{tabular}{|c|c|c|c|c|c|}
\hline & $\left|S_{11}\right|$ & $\left|\mathrm{S}_{21}\right|$ & $\mid \mathrm{S}_{3}$ & $\mathrm{~S}_{4}$ & ${ }_{34}-\angle \mathrm{S}_{24}$ \\
\hline $\begin{array}{l}\text { EM Simulation } \\
\text { Measurement }\end{array}$ & $\bar{\square}$ & $\bar{\theta}$ & -- & $\stackrel{\Delta}{\Delta}$ & $\diamond$ \\
\hline
\end{tabular}

(b)

Fig. 7. $K a$-band CMOS rat-race hybrid. (a) Chip photograph of the prototype. (b) Simulated and measured results of the prototype.

and its equivalent TL network are well documented [29]. As shown in Fig. 7(a), the electrical length between Port 2 and Port 4 is three times as great as the quarter-wavelength and the remaining adjacent ports has the length of one quarter-wavelength. The reference impedance of all four ports is $50.0 \Omega$, and the characteristic impedance of the TLs in the entire rat-race is designed as $70.7 \Omega$ to establish the equal power split and power combination. The CCS TL with $Z_{c}=71.8 \Omega$ is applied to the rat-race realization winding course shown in Fig. 1, and the design is fabricated by standard $0.18-\mu \mathrm{m} 1 \mathrm{P} 6 \mathrm{M}$ CMOS technology. The chip area of the prototype shown in Fig. 7(a) is $420.0 \mu \mathrm{m} \times 540.0 \mu \mathrm{m}$ without the contact pads.

The on-chip performances of the prototype are characterized by conducting the same experimental procedures reported in [23] and are compared to the theoretical data, which is computed by the full-wave HFSS simulations with the circuit layout in Fig. 7(a). Fig. 7(b) shows the composite plots, revealing good agreements between the measurements and HFSS simulations. The transmission coefficients, which are shown in Fig. 7(b) in
TABLE III

$K a$-BAND CMOS RAT-RACE DESIGNS

\begin{tabular}{ccccccc}
\hline \hline & $\begin{array}{c}\text { Loss } \\
(\mathrm{dB})\end{array}$ & $\begin{array}{c}f_{0} \\
(\mathrm{GHz})\end{array}$ & $\begin{array}{c}\mathrm{ARF} \\
(\%)\end{array}$ & $\begin{array}{c}P \\
(\mu \mathrm{m})\end{array}$ & $Q$ & Metal Layer \\
\hline$[23]$ & 5.4 & 30.0 & 97.6 & 20.0 & 6.3 & $\mathrm{M}_{1}, \mathrm{M}_{6}$ \\
This work & 3.8 & 34.3 & 96.9 & 30.0 & 9.4 & $\mathrm{M}_{1}, \mathrm{M}_{2}, \mathrm{M}_{6}$ \\
\hline \hline
\end{tabular}

detail, are less than $4.0 \mathrm{~dB}$ from 34.0 to $38.0 \mathrm{GHz}$, indicating the intrinsic loss of less than $1.0 \mathrm{~dB}$. Additionally, the two transmission coefficients in Fig. 7(b) are -3.94 and $-3.75 \mathrm{~dB}$ at $34.3 \mathrm{GHz}$, showing an amplitude in balance of $0.19 \mathrm{~dB}$. Such results show a nearly equal power distribution at two output ports of the prototype. The measured input return loss from 32.8 to $38.1 \mathrm{GHz}$, illustrated by the curve with hollow squares, is higher than $20.0 \mathrm{~dB}$. The measured isolation from 31.5 to $40.0 \mathrm{GHz}$, illustrated by the curve with hollow triangles, is higher than $20.0 \mathrm{~dB}$ and has a maximum value of $58.0 \mathrm{~dB}$ at $36.3 \mathrm{GHz}$. From 31.0 to $37.0 \mathrm{GHz}$, the phase difference between two output ports is approximately $180^{\circ} \pm 5^{\circ}$. By referring to the calculation of the area reduction factor (ARF) reported in [21, eq. (4)], the prototype in Fig. 7(a), which consisted of $14 \times 18$ unit cells with $P=30.0 \mu \mathrm{m}$, achieved an ARF of $96.9 \%$ at $34.3 \mathrm{GHz}$.

A similar design of the $K a$-band rat-race, realized by incorporating CCS TLs on the standard 0.18- $\mu \mathrm{m}$ 1P6M CMOS technology, was also recently reported in [23]. Table III summarizes these two rat-race hybrid designs for the following discussions. The ARF values shown in Table III are calculated by following the definition of Chen and Tzuang [21]. Two rat-race hybrids are designed at different operating frequencies, and implemented by different CCS TLs. The rat-race design in [23] based on a smaller period of unit cells reveals higher ARF values than those obtained by the design reported herein. This observation confirms the prediction reported in [21, Sec. II-B]. The smaller value of the periodicity $(P)$ resulted in a higher ARF. Furthermore, the ARF is $97.1 \%$, which is only $0.5 \%$ lower than that of [23], when the operating frequency of the rat-race design in Fig. 7(a) are scaled down from 34.3 to $30.0 \mathrm{GHz}$. Moreover, the scaling design, which is theoretically analyzed by performing the full-wave HFSS simulations mentioned above, achieved a transmission loss at $30.0 \mathrm{GHz}$ of $4.2 \mathrm{~dB}$, which is $22.2 \%$ lower than that of the design in [23]. Two different approaches are applied to designing CCS TLs, producing two rat-race hybrids with different performances in terms of loss, area, and ARF. The comparisons summarized above lead to the following observations. Increasing $P$ of the CCS TL enhances the $Q$ factor of the CCS TL. As shown in Fig. 1, since $P$ is the main factor managing the occupying area of the CCS TL, increasing $P$ simultaneously causes $A_{N}$ to increase and ARF to decrease. These observations validate the design guidelines in Table II and the predictions of [21]. Moreover, the rat-race hybrid designs presented here demonstrate that CCS TL can systematically miniaturize the TL-based circuit with predictable electrical performances. The loss of the CCS TL-based circuit is confined to the desired area, and the CCS TL circuit designed by different approaches produced different ARF values for circuit miniaturization. 


\section{CONCLUSION}

The CMOS CCS TL has been reported in detail. By following the universal design guidelines, the CCS TL can be designed with a wide range of characteristic impedance, high SWF, and the satisfaction of the metal density requirement. Additionally, when the physical length is fixed, the ratio of the CCS TL area to its corresponding $Q$ factor approaches a constant and can be applied to estimating the cost of loss for the CMOS circuit miniaturization.

\section{REFERENCES}

[1] C. Cojocaru, T. Pamir, F. Balteanu, A. Namdar, D. Payer, I. Gheorghe, T. Lipan, K. Sheikh, J. Pingot, H. Paananen, M. Littow, M. Cloutier, and E. MacRobbie, "A $43 \mathrm{~mW}$ Bluetooth transceiver with $-91 \mathrm{dBm}$ sensitivity," in Proc. IEEE Solid-State Circuits Conf., 2003, vol. 1, 90-480.

[2] M. Zargari, M. Terrovitis, S. Jen, B. Kaczynski, M. Lee, M. Mack, S. Mehta, S. Mendis, K. Onodera, H. Samavati, W. Si, K. Singh, A. Tabatabaei, D. Weber, D. Su, and B. Wooley, "A single-chip dual-band tri-mode transceiver for IEEE $802.11 \mathrm{a} / \mathrm{b} / \mathrm{g}$ wireless LAN," IEEE $J$. Solid-State Circuits, vol. 39, no. 12, pp. 2239-2249, Dec. 2004.

[3] P. Zhang, L. Der, D. Guo, I. Sever, T. Bourdi, C. Lam, A. Zolfaghari, J. Chen, D. Gambetta, B. Cheng, S. Gowder, S. Hart, L. Huynh, T. Nguyen, and B. Razavi, "A single-chip dual-band direct-conversion IEEE $802.11 \mathrm{a} / \mathrm{b} / \mathrm{g}$ WLAN transceiver in $0.18 \mu \mathrm{m}$ CMOS," IEEE $J$. Solid-State Circuits, vol. 40, no. 9, pp. 1932-1939, Sep. 2005.

[4] T. Maeda, H. Yano, S. Hori, N. Matsuno, T. Yamase, T. Tokairin, R. Walkington, N. Yoshida, K. Numata, K. Yanagisawa, Y. Takahashi, M. Fujii, and H. Hida, "Low-power consumption direction-conversion CMOS transceiver for multistandard $5-\mathrm{GHz}$ wireless LAN systems with channel bandwidths of 5-20 MHz," IEEE J. Solid-State Circuits, vol. 41, no. 2, pp. 357-383, Feb. 2006.

[5] Y. Palaskas, A. Ravi, S. Pellerano, B. R. Carlton, M. A. Elmala, R. Bishop, G. Banerjee, R. B. Nicholls, S. K. Ling, N. Dinur, S. S. Taylor, and K. Soumyanath, "A 5-GHz 108-Mb/s $2 \times 2$ MIMO transceiver RFIC with fully integrated $20.5-\mathrm{dBm} P_{1}$ dB power amplifiers in 90-nm CMOS," IEEE J. Solid-State Circuits, vol. 41, no. 12, pp. 2746-2756, Dec. 2006.

[6] C. Doan, S. Emami, A. Niknejad, and R. Brodersen, "Millimeter-wave CMOS design," IEEE J. Solid-State Circuits, vol. 40, no. 1, pp. 144-155, Jan. 2005.

[7] H. Shigematsu, T. Hirose, F. Brewer, and M. Rodwell, "Millimeterwave CMOS circuit design," IEEE Trans. Microw. Theory Tech., vol. 53, no. 2, pp. 472-477, Feb. 2005.

[8] P.-S. Wu, H.-Y. Chang, M.-D. Tsai, T.-W. Huang, and H. Wang, "New miniature 15-20-GHz continuous-phase amplitude control MMICs using 0.18- $\mu \mathrm{m}$ CMOS technology," IEEE Trans. Microw. Theory Tech., vol. 54, no. 1, pp. 10-19, Jan. 2006.

[9] C.-H. Wang, Y.-H. Cho, C.-S. Lin, H. Wang, C.-H. Chen, D.-C. Niu, J. Yeh, C.-Y. Lee, and J. Chern, "A $60 \mathrm{GHz}$ transmitter with integrated antenna in $0.18 \mu \mathrm{m} \mathrm{SiGe} \mathrm{BiCMOS}$ technology," in Proc. IEEE SolidState Circuits Conf., 2006, pp. 659-668.

[10] C.-M. Lo, C.-S. Lin, and H. Wang, "A Miniature $V$-band 3-stage cascode LNA in $0.13 \mu \mathrm{m}$ CMOS," in IEEE Solid-State Circuits Conf., 2006, pp. 1254-1263.

[11] G. Six, G. Prigent, E. Rius, G. Dambrine, and H. Happy, "Fabrication and characterization of low-loss TFMS on silicon substrate up to 220 GHz," IEEE Trans. Microw. Theory Tech., vol. 53, no. 1, pp. 301-305, Jan. 2005

[12] L. F. Tiemeijer, R. M. T. Pijper, R. J. Havens, and O. Hubert, "Low-loss patterned ground shield interconnect transmission lines in advanced IC processes," IEEE Trans. Microw. Theory Tech., vol. 55, no. 3, pp. 561-570, Mar. 2007.

[13] I. Toyoda, T. Tokumitsu, and M. Aikawa, "Highly integrated threedimensional MMIC single-chip receiver and transmitter," IEEE Trans. Microw. Theory Tech., vol. 44, no. 12, pp. 2340-2346, Dec. 1996.
[14] T. Tokumitsu, M. Hirano, K. Yamasaki, C. Yamaguchi, K. Nichikawa, and M. Aikawa, "Highly integrated three-dimensional MMIC technology applied to novel masterslice GaAs- and Si-MMIC's," IEEE Trans. Microw. Theory Tech., vol. 32, no. 9, pp. 1334-1341, Sep. 1997.

[15] I. Toyoda, K. Nichikawa, T. Tokumitsu, K. Kamogawa, C. Yamaguchi, M. Hirano, and M. Aikawa, "Three-dimensional masterslice MMIC on Si substrate," IEEE Trans. Microw. Theory Tech., vol. 45, no. 12, pp. 2524-2530, Dec. 1997

[16] K. Nichikawa, K. Kamogawa, K. Inoue, K. Onodera, T. Tokumitsu, M. Tanaka, I. Toyoda, and M. Hirano, "Miniaturized millimeter-wave masterslice 3-D MMIC amplifier and mixer," IEEE Trans. Microw. Theory Tech., vol. 47, no. 9, pp. 1856-1862, Sep. 1999.

[17] C. Warns, W. Menzel, and H. Schumacher, "Transmission lines and passive elements for multilayer coplanar circuits on silicon," IEEE Trans. Microw. Theory Tech., vol. 46, no. 5, pp. 616-622, May 1998.

[18] K. Hettak, G. Morin, and M. Stubbs, "The integration of thin-film microstrip and coplanar technologies for reduced-size MMICs," IEEE Trans. Microw. Theory Tech., vol. 53, no. 1, pp. 283-291, Jan. 2005.

[19] M. Chirala and C. Nguyen, "Multilayer design techniques for extremely miniaturized CMOS microwave and millimeter-wave distributed passive circuits," IEEE Trans. Microw. Theory Tech., vol. 54, no. 12 , pp. $4218-4224$, Dec. 2006.

[20] C.-K. C. Tzuang, C.-H. Chang, H.-S. Wu, S. Wang, S.-X. Lee, C.-C. Chen, C.-Y. Hsu, K.-H. Tsai, and J. Chen, "An $X$-band CMOS multifunction-chip FMCW radar," in IEEE MTT-S Int. Microw. Symp. Dig., 2006, pp. 2011-2014.

[21] C.-C. Chen and C.-K. C. Tzuang, "Synthetic quasi-TEM meandered transmission lines for compacted microwave integrated circuits," IEEE Trans. Microw. Theory Tech., vol. 52, no. 6, pp. 1637-1647, Jun. 2004.

[22] C.-K. C. Tzuang, H.-H. Wu, H.-S. Wu, and J. Chen, "CMOS active bandpass filter using compacted synthetic quasi-TEM lines at $C$-band," IEEE Trans. Microw. Theory Tech., vol. 54, no. 12, pp. 4548-4555, Dec. 2006.

[23] S. Wang and C.-K. C. Tzuang, "Compacted $K a$-band CMOS rat-race hybrid using synthesized transmission lines," in IEEE MTT-S Int. Microw. Symp. Dig., 2007, pp. 1023-1026.

[24] A. B. Kahng, G. Robins, A. Singh, and A. Zelikovsky, "New and exact filling algorithms for layout density control," in Proc. 12th Int. VLSI Design Conf., Jan. 1999, pp. 106-110.

[25] T. Itoh, "Generalized spectral domain method for multiconductor printed lines and its applications to tunable suspended microstrips," IEEE Trans. Microw. Theory Tech., vol. MTT-26, no. 12, pp. 983-987, Dec. 1978.

[26] H. Kamitsuna, "A very small, low-loss MMIC rat-race hybrid using elevated coplanar waveguides," IEEE Microw. Guided Wave Lett., vol. 2, no. 8, pp. 337-339, Aug. 1992.

[27] G. E. Ponchak and A. N. Downey, "Characterization of thin film microstrip lines on polyimide," IEEE Trans. Compon., Packag., Manuf. Technol. B, vol. 21, no. 2, pp. 171-176, May 1998.

[28] F. Schnieder and W. Heinrich, "Model of thin-film microstrip line for circuit design," IEEE Trans. Microw. Theory Tech., vol. 49, no. 1, pp. 104-110, Jan. 2004.

[29] R. E. Collin, Foundations for Microwave Engineering, 2nd ed. New York: McGraw-Hill, 1992.

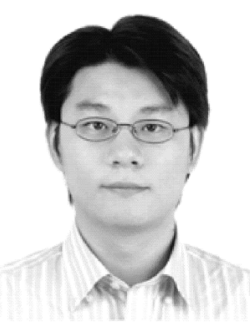

Meng-Ju Chiang (S'05) received the B.S. degree in electrical engineering from Fong-Chia University, Taichung, Taiwan, R.O.C., in 2002, the M.S. degree in electronic engineering from the Chung-Cheng Institute of Technology, National Defense University, Taoyuang, Taiwan, R.O.C., in 2004, and is currently working toward the Ph.D. degree at the Graduate Institute of Communication Engineering, National Taiwan University, Taipei, Taiwan, R.O.C.

His research interests include the design of microwave/millimeter-wave passive circuits and antenna designs, CMOS RF integrated circuits, and development of advanced guiding structures for CMOS RF SOC. 


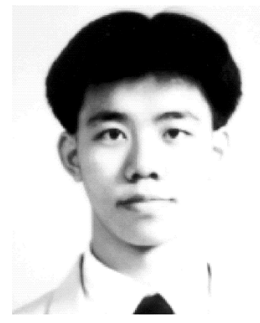

Hsien-Shun Wu (S'97-M'05) received the B.S. degree in electronic engineering from the National Taipei University of Technology, Taipei, Taiwan, R.O.C. in 1999, and the M.S. and Ph.D. degrees in communication engineering from National Chiao Tung University, Hsinchu, Taiwan, R.O.C. in 2001, and 2005 , respectively.

He is currently a Post-Doctoral Research Fellow with the Graduate Institute of Communication Engineering, National Taiwan University, Taipei, Taiwan, R.O.C. His research interests include the design of wireless system modules and the development of synthetic waveguides for RF circuits.

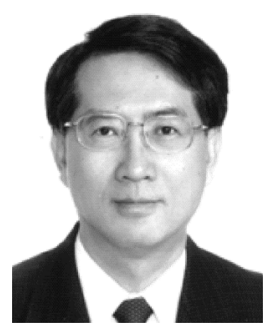

Ching-Kuang C. Tzuang (S'80-M'80-SM'92F'99) received the B.S. degree in electronic engineering from National Chiao Tung University, Hsinchu, Taiwan, R.O.C., in 1977, the M.S. degree from the University of California at Los Angeles (UCLA), in 1980, and the Ph.D. degree in electrical engineering from the University of Texas at Austin, in 1986.

From 1981 to 1984, he was with TRW, Redondo Beach, CA, where he was involved with analog and digital MMICs. From 1986 to 2004, he was with the Institute of Communication Engineering, National Chiao Tung University. In February 2004, he joined the Graduate Institute of Communication Engineering, Department of Electrical Engineering, National Taiwan University, Taipei, Taiwan, R.O.C., where he conducts research on advanced guiding structures for research and development of RF SOC and integrating active and passive microwave/millimeter-wave RF signal-processing components into a single chip. He has consulted the Wireless Communications Engineering Center (WiCE) on RF SOC/system-in-package (SIP). His research activities also involve the design and development of millimeter-wave and microwave active and passive circuits and the field theory analysis and design of various complex waveguide structures and large-array antennas. He has supervised 66 M.S. students and $23 \mathrm{Ph} . \mathrm{D}$. students.

Dr. Tzuang helped formed the IEEE Microwave Theory and Techniques Society (IEEE MTT-S) Taipei Chapter, and served as secretary, vice chairman, and chairman in 1988, 1989, and 1990, respectively. Since 2004, he has assisted the Taiwan Electrical and Electronic Manufacturers' Association (TEEMA), promoting standardization and application of millimeter-wave technology. 